\title{
A Novel Deletion in the RPL5 Gene in a Lebanese Child With Diamond Blackfan Anemia Unresponsive to Steroid Treatment
}

Roula A. Farah, MD, FAAP, Lojine Kamel, MD, Noemi Roy, MD, Melanie Proven, MD, $\ddagger$ Katherine Wray, MD, $\ddagger$ Irene Roberts, MD, and Marcin W. Wlodarski, MD

Diamond-Blackfan Anemia (DBA), a rare inherited form of pure red cell aplasia, usually presents shortly after birth or in the first year of life. Typically, the anemia is normocytic or macrocytic and the bone marrow shows selective erythroblastopenia with normal numbers and maturation of other lineages. In $\sim 40 \%$ of cases, affected individuals have associated growth retardation, craniofacial malformation, upper limb abnormalities, urinary system malformations, and/or cardiac defects. Most patients require treatment to maintain hemoglobin at sufficient levels for normal growth and well-being, either with low-dose steroids or regular red blood cell transfusions. The only known curative treatment is hematopoietic stem cell transplantation (HSCT). 1

The genetic cause of DBA is known in approximately two-thirds of patients. 2 It is caused by loss-of-function aberrations in ribosomal protein (RP) genes resulting in defective ribosome biogenesis. 3 To date, mutations or whole gene deletions in over 20 RP genes have been identified.2 RPS19 and RPL5 are the most commonly affected genes, accounting for about $25 \%$ and $6.6 \%$ of cases, respectively.4,5 Here we report a 15-year-old Lebanese girl diagnosed with DBA in infancy and found to have a novel heterozygous mutation in RPL5. The mutation is a 9 nucleotide deletion spanning the exon 4-intron 4 junction, predicted to generate a null allele of RPL5.

\section{CASE SUMMARY AND DISCUSSION}

The patient, a native of the Bekaa valley in Lebanon, presented at the age of 25 days with increasing pallor, poor weight gain, and severe macrocytic anemia. Complete blood count (CBC) showed Hb of $32 \mathrm{~g} / \mathrm{L}, \mathrm{MCV}$ of $101 \mathrm{fL}, \mathrm{WBC}$ of $4 \times 109$ /L, platelet count of $153 \times 103 / \mu \mathrm{L}$. Her parents are healthy first-degree cousins with normal CBCs and peripheral smears. The patient has an older brother and younger sister, both of whom are healthy and there is no family history of anemia. The patient was initially treated by red blood cell transfusions and was referred to our clinic at the age of 9 months, where she was noted to have the "classic DBA face" initially described by Cathie6 with short wide nose, low set ears, cupped ears, and hypertelorism (Fig. 1). She had growth delay but no thumb or hand anomalies. The peripheral smear showed mild anisocytosis and the bone marrow (BM) aspirate and core biopsy were normocellular with severe erythroid hypoplasia, consistent with DBA. The BM karyotype and Mitomycin C-induced chromosomal breakage were normal.

The patient did not respond to 3 prednisone courses administered at age of 5 months, 10 months, and 2 years. In between the steroid trials she received regular transfusions, and starting age 2 years 9 months she was placed on a chronic transfusion program. Iron chelation by subcutaneous injections of Deferoxamine, 6 days per week, was initiated at the age of 3 years and 8 months and switched to oral Deferasirox at the age of 9 years and 3 months. MRI-based or SQUID-based assessment of iron overload was not accessible to the patient. Growth hormone therapy had been administered for short stature at the age of 7 years. While growth 
hormones have been successfully used in DBA,7,8 it has to be cautiously noted that DBA can also be considered a cancer predisposition syndrome9,10 and it is not clear if the use of growth hormones could potentially increase the risk of cancer in DBA patients.

At the age of 15 years, while being considered for HSCT, targeted resequencing as previously described11 using peripheral blood DNA was carried out to determine the molecular basis of her disease and to allow for genetic testing of her clinically unaffected matched sibling donor. A novel heterozygous frameshift mutation in the RPL5 gene (exon 4, c.320_324+4delGCAGGGTAT) was found. At the mRNA level, this mutation was predicted to disrupt the exon-intron border and abolish splicing. The parental DNA was tested by Sanger sequencing and both parents were shown to be wild-type at the site of this deletion, confirming that the child's mutation was de novo (Fig. 2).

Heterozygous mutations in RP genes have been described in association with DBA, most commonly in RPS19 and, as in our case, in RPL5.5 Patients with RPL5 mutations are reported to have multiple physical abnormalities, including craniofacial defects, heart defects and thumb abnormalities, although these were not present in our patient.5 The molecular basis of DBA in our patient was not determined until she was in her teens. However, establishing the genetic cause is helpful for family counseling and HSCT donor selection. While the pathogenicity of this variant was not formally confirmed by functional assays, and the targeted sequencing panel included only the 9 RP genes with known pathogenic variants, 11 the disruption of the exon 4intron 4 junction of RPL5 with a 9 nucleotide deletion is a very strong candidate mutation for the molecular basis of DBA in this case. On the basis of the ExAC population database, RPL5 gene is very intolerant to loss of function mutations, with 15 expected and 0 observed variants and a pLI score of 0.99 (exac. broadinstitute.org). Furthermore, the majority of RPL5 mutations reported in patients are null mutations.13

At the age of 15 years the patient underwent HSCT from her HLA-matched sibling donor, after myeloablative conditioning using busulfan and cyclophosphamide. She died from transplantrelated complications 7 weeks later (day+55). She developed Graft versus Host Disease, veno-occlusive disease of the liver, and septic shock due to Pseudomonas aeruginosa infection. Although her serum ferritin was relatively low (956 ng/mL) at the time of HSCT, organ iron assessment was not available and therefore it is not clear if transfusion-related iron overload contributed to these complications.14 It is possible that her outcome would have been better if she had been transplanted earlier (as recommended by an international consensus group in inherited BM failure syndromes15), however this could not be accomplished due to financial limitations. For the best possible outcome HSCT should ideally be performed before the age of 10 years.16 The results of HSCT for DBA performed in recent years indicate that while choice of donor (matched sibling vs. matched unrelated donor) leads to similar HSCT outcomes, recipient age is a negative prognostic factor.17-19

\section{CONCLUSIONS}

This case illustrates several important problems arising from difficulties in clinical management in a population of patients with limited resources. For instance, 
accurate evaluation of iron overload by organ iron assessment, and not ferritin measurements alone, was not accessible. DBA patients undergoing chronic transfusions can load organ iron quickly and ferritin measurements alone may not reflect this.20 It is possible that the patient described had severe iron overload despite medium to low ferritin levels. In addition, it is important to assess the risk to benefit before deciding for HSCT. Age and iron overload have been discussed as risk factors in HSCT outcome.17-19 This patient died from multiorgan failure following veno-occlusive disease, which might have been contributed to by iron overload. Finally, HSCT should ideally have been performed timely after the failed steroid trials but was delayed due to financial constraints.

Easier access to transplantation, early and aggressive iron chelation, and national or international programs supporting the financial burden of these procedures should improve the outcome for children in all countries. 